\title{
Adaptive Multiple-Antenna Receiver for CDMA Mobile Reception
}

\author{
Stefan Werner and Timo Laakso \\ Helsinki University of Technology \\ Laboratory of Telecommunications Technology \\ email: Stefan.Werner@hut.fi, Timo.Laakso@hut.fi \\ Jorma Lilleberg \\ Nokia Mobile Phones \\ email: Jorma.Lilleberg@nmp.nokia.com
}

\begin{abstract}
This paper examines the utilization of multiple antennas for Direct-Sequence Code Division Multiple Access (DS-CDMA) mobile communications at the mobile receiver. A linear singleuser multiple-antenna receiver is derived and its performance is compared with that of the conventional matched filter. An adaptive implementation of the receiver is also considered. The results show that the multiple-antenna receiver is insensitive to the interfering powers and can provide room for more users or a smaller number of antennas than the matched filter solution. Using the adaptive algorithm, the performance even with a single antenna is often much better than that of a matched filter with 4 antennas.
\end{abstract}

\section{INTRODUCTION}

In a CDMA cellular mobile communication system, the signal reception at the mobile unit differs essentially from that at the base station. First, the desired signal and all the same-cell interference comes from the base station from the same direction and through the same channel. The transmission can also usually be assumed to be synchronized so that all the symbol periods overlap exactly. Second, the signal processing should be as simple as possible due to the strict constraints for price, complexity, power consumption, and physical size of the mobile receiver.

For utilization of multiple antennas at the mobile unit this means that the concept of beamforming is not necessary as most of the interference comes from exactly the same direction as the desired signal. Furthermore, beamforming will not be easily implemented, as it is difficult to attach many antenna elements at the receiver. A mobile phone handset can hardly bear more than two antennas; on the top of a car roof or a laptop computer one can probably put at most five elements. Hence, multiple antennas at the mobile receiver are more useful for providing diversity against additive noise and fading.

In this paper we investigate the use of multiple antennas at the mobile direct-sequence CDMA receiver using multiple antennas. The basic goal is to utilize the antenna output signals in order to maximize signal-to interference and noise ratio. For interference suppression we assume knowledge of the desired signal's waveform. In [1] a stochastic gradient algorithm was proposed only requiring knowledge of the desired user's spreading code. This approach was further investigated in [2] where a structure similar to the generalized sidelobe canceller [3] used in array processing was implemented to get a more robust implementation of the blind algorithm of [1]. Here we generalize the ideas from [1] to include multiple antennas and employ an adaptive algorithm.

The paper is organized as follows. In Section II, we construct a signal model for a downlink transmission system with multiple antenna elements at the mobile receiver. In Section III we derive a linear single-user multiple-antenna receiver for a stationary channel. In Section IV, an LMS-type adaptive algorithm is considered, which is a generalization of that presented in [1]. The performance of the receiver and its adaptive implementation is demonstrated with a simplified example in Section $\mathrm{V}$, followed by conclusions.

\section{SYSTEM MODEL}

In downlink transmission (base station to mobile), signals associated with a number of simultaneously active users are transmitted over the same mobile channel. A mobile receiver, equipped with $N$ antennas will receive the transmitted signal over $N$ different channels. Fig. 1 shows the block diagram of the system.

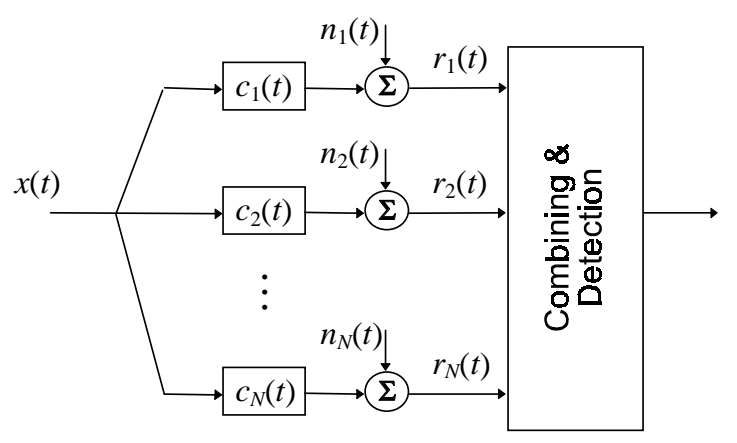

Figure 1: Block diagram of the communication system.

The system under consideration consists of $K$ users transmitting information with binary antipodal signals, $b_{k}^{(m)} \in\{-$ $1,1\} k=1,2, \ldots, K$, with bit duration $T_{b}$. The data stream of the $k^{\text {th }}$ user is spread by multiplying the data with a pseudorandom code sequence to form the signal: 


$$
x_{k}(t)=\sum_{m=-\infty}^{\infty} b_{k}^{(m)} s_{k}\left(t-m T_{b}\right)
$$

The code sequence has the form:

$$
s_{k}(t)=\sum_{j=1}^{G} s_{k}^{(j)} p\left(t-j T_{c}\right)
$$

where $G=T_{b} / T_{c}$ is the number of chips per bit, $\mathrm{s}_{k}^{(j)} \in\{-1,1\}$, and $p(t)$ is the chip waveform. The chip waveform is here assumed to be a rectangular pulse with unit energy and duration $T_{b}$, i.e., $s_{k}(t)=0$ for $t \notin\left[0, T_{b}\right]$. The continuous transmitted signal is obtained as:

$$
x(t)=\sum_{k=1}^{K} \sqrt{2} A_{k} x_{k}(t) \cos \left(\omega_{c} t+\phi\right)
$$

where $A_{k}$ the amplitude of the $k^{\text {th }}$ user, $\omega_{c}$ is the carrier frequency and $\phi$ is the carrier phase. In the case of an AWGN channel the received signal at the $i^{\text {th }}$ antenna can be written as

$$
r_{i}(t)=\operatorname{Re}\left\{\sum_{k=1}^{K} \sqrt{2} A_{k} x_{k}\left(t-\tau_{i}\right) e^{j\left(\omega_{c} t+\phi-\omega_{c} \tau_{i}\right)}\right\}+n_{i}(t)
$$

where $\tau_{i}$ is the propagation delay of the incoming signal at antenna number $i$. and $n_{i}(t)$ represents the AWGN with two sided spectral density $N_{0} / 2[\mathrm{~W} / \mathrm{Hz}]$ at antenna number $i$. The noise at one antenna element is assumed to be independent from those at the others. In front of every antenna is an I-Q stage followed by a chip-matched filter (integrate and dump filter with integration time $T_{c}$ ). If the samples from chipmatched filter during the $m^{\text {th }}$ bit interval are collected in the vectors

$$
\begin{aligned}
& \mathbf{r}_{i}(m)=\left[r_{i}(m G+1) r_{i}(m G+2) \cdots r_{i}(m G+G)\right]^{\mathrm{T}} \\
& \mathbf{n}_{i}(m)=\left[n_{i}(m G+1) n_{i}(m G+2) \cdots n_{i}(m G+G)\right]^{\mathrm{T}}
\end{aligned}
$$

we can write the received discrete-time signal as:

$$
\mathbf{r}_{i}(m)=\mathbf{S}_{i} \mathbf{A}_{i} \mathbf{b}(m)+\mathbf{n}_{i}(m)
$$

where $\mathbf{S}_{i}$ is the $G \times K$ spreading matrix containing the spreading sequences for the different users:

$$
\mathbf{S}_{i}=\left[\mathbf{s}_{1 i} \mathbf{S}_{2 i} \ldots \mathbf{S}_{k, i}\right]
$$

where $\mathbf{s}_{k, i}$ is the discrete-time delayed version of the $k^{\text {th }}$ user's sampled code sequence at antenna $i, \mathbf{A}_{i}$ is a diagonal amplitude matrix of the form:

$$
\mathbf{A}_{i}=\operatorname{diag}\left[a_{i} A_{1} a_{i} A_{2} \cdots a_{i} A_{k}\right]
$$

where $a_{i}=e^{j(\phi-\omega \tau)}$ is the complex phase factor at the $i^{\text {th }}$ antenna. Finally, $\mathbf{b}(m)$ is a vector containing the transmitted bits of the users:

$$
\mathbf{b}^{(m)}=\left[\begin{array}{lll}
b_{1}^{(m)} & b_{2}^{(m)} \ldots b_{K}^{(m)}
\end{array}\right]^{\mathrm{T}}
$$

The noise sequence is independent between the antennas so that:

$$
E\left\{\mathbf{n}_{i}(m) \mathbf{n}_{j}^{\mathrm{H}}(m)\right\}=\sigma_{i}^{2} \mathbf{I}_{G} \delta(i-j)
$$

If the antennas are spaced close together, the sampled code sequences will be the same in all antennas. In the case of an $\mathrm{N}$ element linear array, the phase factor is given by [4]

$$
a_{i}=\exp \left[j\left(\phi-2 \pi(i-1) \frac{\Delta}{\lambda_{c}} \sin \theta\right)\right]
$$

where $\Delta$ is the element spacing, $\lambda_{c}$ is the wavelength of the carrier and $\theta$ is the direction of the incoming signal with respect to the array normal. The optimal Maximum Likelihood detector could now be derived. However, this detector is complex and requires of every user's signature sequence, which cannot be assumed to be known at the mobile receiver. Therefore, in the next section we derive a linear solution suited for a mobile receiver.

\section{LINEAR DETECTOR}

In this section, a linear single-user multi-antenna (LSUMA) detector is derived. The detector assumes knowledge of the timing and carrier phase in every antenna. The structure of the receiver equipped with $N$ antennas is shown in Fig. 2. Each of the $N$ antenna branches contains a linear filter whose coefficients are to be optimized. The filtered signals from each antenna are then added together to form a decision variable. In Fig. 2, $\mathbf{r}_{i}$ denotes the received signal after chip-matched filtering at antenna $i, \mathbf{h}_{i}$ contains the complex filter coefficients for the $i^{\text {th }}$ antenna, and $z$ is the decision variable formed by adding the filtered outputs from each antenna.

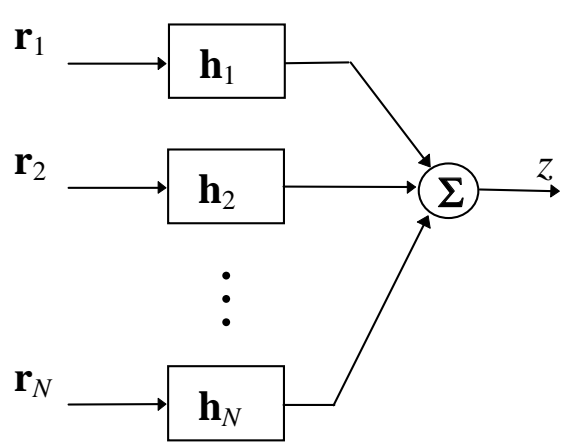

Figure 2: Structure of linear detector

In order to get a compact notation, let us collect the filter coefficients and the received sequences from the antennas in vectors as

$$
\begin{gathered}
\mathbf{h}=\left[\begin{array}{lll}
\mathbf{h}_{1}^{\mathrm{T}} & \ldots & \mathbf{h}_{N}^{\mathrm{T}}
\end{array}\right]^{\mathrm{T}} \\
\mathbf{r}=\left[\begin{array}{llll}
\mathbf{r}_{1}^{\mathrm{T}} & \mathbf{r}_{2}^{\mathrm{T}} & \ldots & \mathbf{r}_{N}^{\mathrm{T}}
\end{array}\right]^{\mathrm{T}}
\end{gathered}
$$

Using the above notation, the output from the receiver can be written as

$$
z=\mathbf{h}^{\mathrm{H}} \mathbf{r}
$$

The variance of the output, i.e., the output power, is

$$
E\left\{|z|^{2}\right\}=E\left\{\mathbf{h}^{\mathrm{H}} \mathbf{r r}^{\mathrm{H}} \mathbf{h}\right\}=\mathbf{h}^{\mathrm{H}} \mathbf{R} \mathbf{h}
$$


where $\mathbf{R}$ is the correlation matrix with elements

$$
\mathbf{R}_{i j}=E\left\{\mathbf{r}_{i} \mathbf{r}_{j}^{\mathrm{H}}\right\}=\mathbf{S}_{i} E\left\{\mathbf{A}_{i} \mathbf{A}_{j}^{\mathrm{H}}\right\} \mathbf{S}_{j}^{\mathrm{H}}+\sigma_{i} \mathbf{I}_{G} \boldsymbol{\delta}(i-j)
$$

The first term in (25) describes the correlation between the amplitudes of received signal components between antenna $i$ and $j$.

Let us now state the optimization problem as follows. We want to find the filters $\mathbf{h}_{i}$ such that the output variance of (19) is minimized under the constraint that the desired user's code sequence in every antenna can pass with unity response. In order to formulate this in a compact form we introduce the $G N \times N$ matrix $\mathbf{C}$ and the $N \times 1$ vector $\mathbf{u}$ as

$$
\begin{gathered}
\mathbf{C}=\left[\begin{array}{cccc}
a_{1} \mathbf{s}_{1,1} & 0 & \ldots & 0 \\
0 & a_{2} \mathbf{s}_{1,2} & 0 & \vdots \\
\vdots & 0 & \ddots & 0 \\
0 & \ldots & 0 & a_{N} \mathbf{s}_{1, N}
\end{array}\right] \\
\mathbf{u}=\left[\left|a_{1}\right|^{2}\left|a_{2}\right|^{2} \ldots\left|a_{N}\right|^{2}\right]^{\mathrm{T}}
\end{gathered}
$$

where $\mathbf{s}_{1, i}$ is the code sequence and $a_{i}$ is the complex phase factor of the desired user at the $i^{\text {th }}$ antenna element. The minimization problem can now be formulated as

$$
\begin{aligned}
& \hat{\mathbf{h}}=\arg \min _{\mathbf{h}} E\left\{|z|^{2}\right\} \\
& \text { subject to: } \quad \mathbf{C}^{\mathrm{H}} \mathbf{h}=\mathbf{u}
\end{aligned}
$$

The formulation in Eq. (23) is general in the sense that if the interference environment changes, Eq. (23) remains the same. The solution to this problem is found by the method of Lagrange multipliers, see, e.g., [5]

$$
\mathbf{h}_{\text {opt }}=\mathbf{R}^{-1} \mathbf{C}\left[\mathbf{C}^{\mathrm{H}} \mathbf{R}^{-1} \mathbf{C}\right]^{-1} \mathbf{u}
$$

If the antennas are spaced closely together, we will sample the same code sequence in every antenna branch. In that case the different antenna filters $\mathbf{h}_{\mathrm{i}}$ would be the same except for the phase factors. An equivalent structure with considerably reduced complexity can be used by first combine the different antenna elements before the interference suppression. This reduced complexity solution is, however, not valid in a more general setting.

The minimum output variance is obtained by substituting (24) into (19):

$$
E\left\{|z|^{2}\right\}=\mathbf{u}^{\mathrm{H}}\left[\mathbf{C}^{\mathrm{H}} \mathbf{R}^{-1} \mathbf{C}\right]^{-1} \mathbf{u}
$$

The closed-form solution is not suitable for practice, as we would need to estimate the correlation matrix and invert it every time the channel changes. In the next section an adaptive implementation of the detector is considered.

\section{ADAPTIVE ALGORITHM}

In this section, we study a simple adaptive implementation of Eq. (24). The algorithm was proposed by Frost [6], and it was originally derived for adaptive array processing. It is an LMStype algorithm and the idea is to ensure the constraints at each iteration. The constrained algorithm can be written as

$$
\begin{gathered}
\mathbf{h}(0)=\mathbf{f} \\
\left.\mathbf{h}(m+1)=\mathbf{P}\left[\mathbf{h}(m)-\mu z^{*}(m) \mathbf{r}(m)\right)\right]+\mathbf{f}
\end{gathered}
$$

where $\mu$ is the step size parameter, and $\mathbf{P}$ and $\mathbf{f}$ are defined as

$$
\begin{gathered}
\mathbf{P}=\mathbf{I}-\mathbf{C}\left(\mathbf{C}^{\mathrm{H}} \mathbf{C}\right)^{-1} \mathbf{C}^{\mathrm{H}} \\
\mathbf{f}=\mathbf{C}\left(\mathbf{C}^{\mathrm{H}} \mathbf{C}\right)^{-1} \mathbf{u}
\end{gathered}
$$

It is easy to check that the initial value, $\mathbf{h}(0)$, in Eq. (28) satisfies the constraints in Eq. (24). The expression inside the brackets of Eq. (29) is the unconstrained LMS update of the weight vector. In general, this update does not lie in the constraint plane. In order to move the unconstrained update back onto the constraint plane, it is first projected onto the constraint subspace by the matrix $\mathbf{P}$, i.e., all components perpendicular to the plane $\mathbf{C}^{\mathrm{H}} \mathbf{h}=0$ are removed. Finally, the vector is moved back to the constraint plane by adding the vector f. The updated weight vector now satisfies the constraints within the numerical precision used in the implementation.

\section{NUMERICAL RESULTS}

In this section, we study the optimal performance and the adaptive implementation of the linear single-user multipleantenna (LSUMA) receiver. The antennas are structured as a uniform linear array (ULA) with half the wavelength spacing. The expression for the optimal weight vector in Section III can be calculated by assuming that the spreading codes for the different users and the noise variances at the antennas are known. The performance of the receiver is compared with that of the matched filter solution for multiple antennas. The spreading sequences are Gold codes of length 15 [7].

\section{A. Steady-state performance}

The matched-filter solution maximizes the output SNR in a single-user system and therefore it neglects the presence of other users. Consequently, the performance will be worse when introducing more than one user in the system. Moreover, if the multiple-access interference (MAI) dominates over the noise, adding more antennas will only increase slightly the output SIR. The LSUMA is optimized so that it considers both noise and MAI. Therefore, for a high noise level and low MAI, it tries to average the noise away and if the MAI dominates, the LSUMA concentrates to suppress that instead of the noise. In order to illustrate the behavior described above we plot the output SIR for different levels of MAI to see the performance of the matched filter and the LSUMA. 


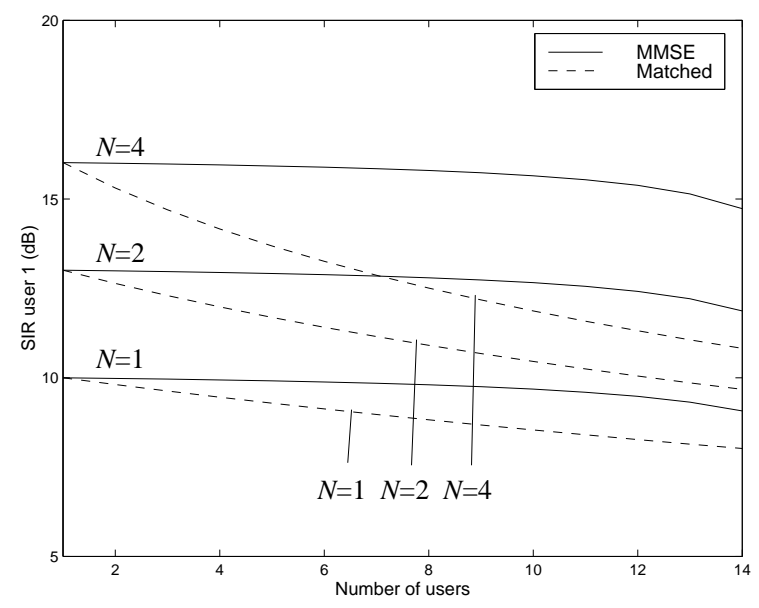

Figure 3: SIR as a function of the number of users for the matched filter (dashed) and the LSUMA (solid), $S N R_{1, i}=10 \mathrm{~dB}, A_{\mathrm{k}}=A_{1}=1$. The number of antennas is one, two and four $(N=1,2,4)$.

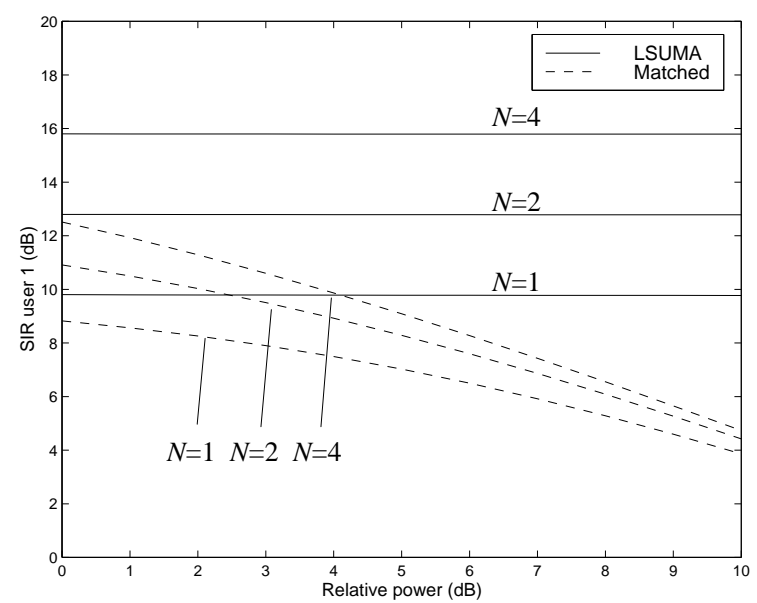

Figure 4: SIR as a function of the relative powers of the undesired users in a system with $\mathbf{8}$ users. The matched filter (dashed) and the LSUMA (solid), $S N R_{1, i}=10 \mathrm{~dB}$. The number of antennas is one, two and four $(N=1,2,4)$.

In Fig. 3, the SIR as a function of the number of users is shown. The signal-to-noise ratio at the antennas for the desired user is set $S N R_{1, i}=10 \mathrm{~dB}$. The base station transmits with same power to all the users, here set to unity, i.e., $A_{k}=A_{1}=1$. The number of antennas used is one, two, and four.

Fig. 3 clearly shows the degradation of the performance of the matched filter solution as the MAI increases. The performance of the LSUMA is however unaffected and gives a $3 \mathrm{~dB}$ improvement when doubling the number of antennas. Fig. 3 illustrates the fact that, when the MAI is the dominating interference, the increase in the output SIR for the matched filter is small when adding more antennas. The number of users allowed for a fixed SIR antennas is considerably higher for the LSUMA than for the matched filter solution. Furthermore, the same number of users might be served with a smaller number of antennas with the LSUMA (two antennas instead of four).

Let us then take a look at how sensitive the receivers are to changes in the interfering power. We let the powers of the interfering users change with respect to the desired user's power. Fig. 4 shows the optimal SIR as a function of the relative interference power in a system with 8 users. The signal-to-noise ratio at the antennas for the desired user is fixed at $10 \mathrm{~dB}$.

As can be seen from Fig. 4, the LSUMA is insensitive to the relative powers of the interfering users and is able to suppress the strong interfering signals. In contrast, the performance of the matched filter deteriorates drastically for increasing interfering power. The multiple-antenna gain for the matched filter solutions as the MAI increases.

\section{B. Adaptive implementation}

We now consider the implementation of the adaptive algorithm. We study the cases with one, two and four antennas.

In Fig. 5, the SIR versus the number of iterations is shown for the case of eight users where the interfering signals transmit with $10 \mathrm{~dB}$ higher power than the desired user. The performance measure used is the SIR as a function of the number of iterations made by the algorithm. The results are averaged over 500 independent simulations (smoothened). The step sizes used are: $\mu=5 \cdot 10^{-4}$ for one antenna, $\mu=2 \cdot 10^{-4}$ for two antennas, and $\mu=10^{-4}$ for four antennas. In the plots the horizontal dashed lines are the optimum SIR values and the solid lines correspond to the matched filter solutions.

For one antenna, the algorithm converges to its steady-state solution after approximately 800 iterations. For two antennas it takes about 1800 iterations to converge. In the case of four antennas, it takes over 2200 iterations before the steady-state value is reached. In all cases, the initial values are the matched filter solutions. As can be seen from Fig. 5, the differences for the matched filter solutions are small. Faster convergence can be provided at the expense of a lower steady-state value. As Fig. 5 indicate, faster convergence to a fixed level can be increased by adding more antennas. However this requires that the step size is sufficiently large.

Now we plot some bit error (BER) curves after the convergence of the filter. The curves are obtained by averaging 40000 bits from 50 independent trials. The code sequences are the same as previously. In order to reduce the simulation time, the SNR for the desired user is set to $2 \mathrm{~dB}$ at every antenna. The system still contains 8 users.

Fig. 6 shows the BER as a function of the relative power of the interfering users. In the plots the solid lines correspond to the LSUMA receiver and the dashed lines are the matched filter solutions. From the figure we can clearly see that the matched filter solutions tends to the same BER value as the relative power increases. However, the LSUMA is not affected by the increase of the interfering powers., which is in accordance with the SIR plots.

Fig. 7 shows the influence of different choices of step-sizes. All the interfering users transmit with $10 \mathrm{~dB}$ higher power. The dash-dotted curves are the optimum values and the solid lines are the curves obtained by varying the step-size. 


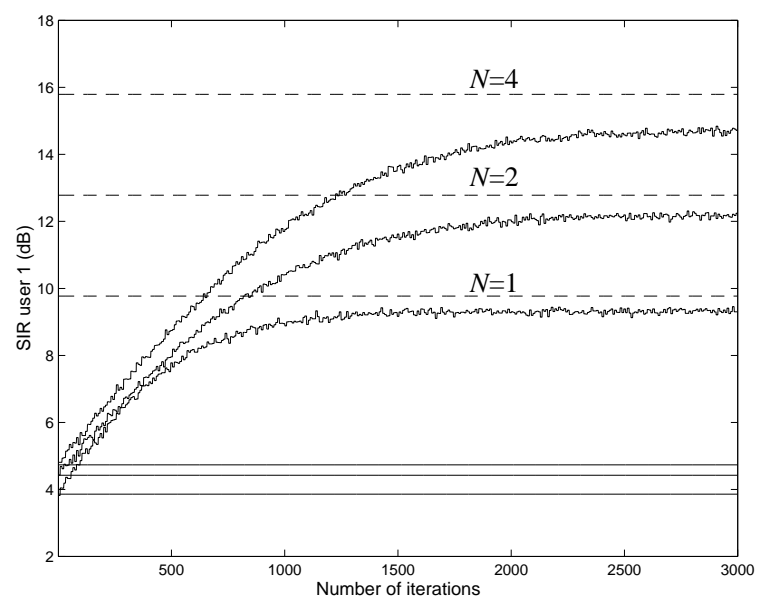

Figure 5:SIR as a function of the number of iterations with Frost algorithm in a system with eight users. The number of antennas is one, two and four $(N=1,2,4)$.

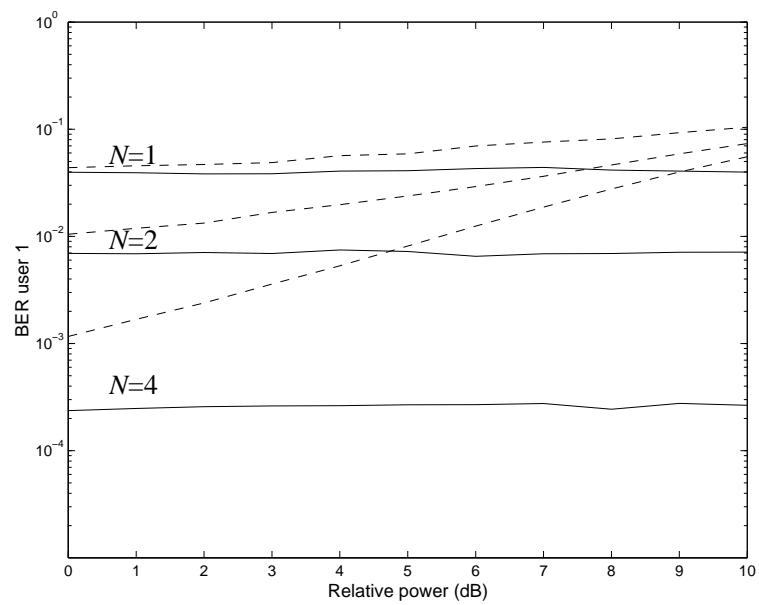

Figure 6: BER as a function of the relative powers of the undesired users in a system with 8 users. The matched filter (dashed) and the LSUMA (solid), $S N R_{1, i}=2 d B$. The number of antennas is one, two and four $(N=1,2,4)$.

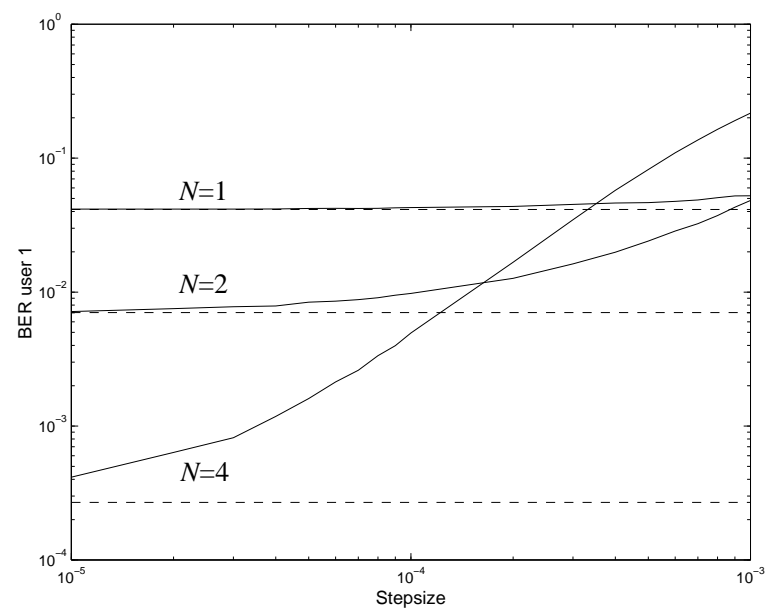

Figure 7: BER as a function of the step size of the adaptive algorithm in a system with 8 users. The optimum values (dash) and the LSUMA (solid), $S N R_{1, i}=2 d B$. The number of antennas is one, two and four $(N=1,2,4)$.
As can be seen from Fig. 7, the choice of the step size has a major impact on the results. As the stepsize becomes larger, the misadjustment and consequently the BER increases. On the other hand, the convergence speed increases. For example, four antennas and a stepsize $\mu=10^{-5}$ takes 2000 iterations to converge to the steady-state BER in the figure. If the stepsize is increased to $\mu=1 \cdot 10^{-4}$ it takes about 200 iterations to reach the steady-state BER.

\section{CONCLUSIONS}

In this paper, we have presented a DS-CDMA multi-antenna receiver suitable for a mobile terminal. An adaptive version of the receiver was also considered. The results show that the linear receiver overcomes the problems associated with matched filter, that is, it suppresses multiple-user interference efficiently and is insensitive to changes in the interfering users' powers. By doubling the number of antennas a $3 \mathrm{~dB}$ gain is achieved. In the matched filter the improvement depends on the number of interfering users and their powers. For a large number of users or high interfering powers the improvement achieved with matched filter becomes negligible. The Frost algorithm is attractive due to its computational simplicity. However, the performance is highly dependent of the step size used. To overcome this problem, other types of algorithms can be used at the expense of computational complexity. Present work includes implementations of more suitable adaptive solutions with a small additional complexity.

\section{ACKNOWLEDGMENT}

This work is part of a research project of the Institute of Radio Communication (IRC) funded by Technology Development Center (TEKES), NOKIA Research Center, Telecom Finland and the Helsinki Telephone Company.

\section{REFERENCES}

[1] M. Honig, U. Madhow, S. Verdú, "Blind adaptive multiuser detection", IEEE Trans. Inform. Theory., vol. 41, pp. 944-960, July 1995.

[2] J. B. Schodorf, D. B. Williams, "A blind adaptive interference cancellation scheme for CDMA systems", Proc. Asilomar Conf. Sig., Sys. and Comp., 1995.

[3] L. J. Griffiths and C. W. Jim, "An alternative approach to linearly constrained beamforming," IEEE Trans. Antennas and Propagation, vol. AP-30, pp. 27-34, Jan. 1982.

[4] J. Litva and T. Kwok-Yeung Lo, Digital Beamforming in Wireless Communications, Artech House, Boston, 1996.

[5] S. Haykin, Adaptive Filter Theory, Prentice Hall, Upper Saddle River, New, Jersey, 1991.

[6] O. L. Frost, III, "An algorithm for linearly constrained adaptive array processing”, Proc. IEEE, vol. 60, pp. 926-935, Aug. 1972.

[7] C. Schlegel, S. Roy, P. D. Alexander and Z.-J. Xiang, "Multiuser projection receivers," IEEE J. Select. Areas Comm., vol. 14, pp. 1610-1618, Oct. 1996. 